TP-TSPU-2/98

TP-TSU-7/98

hep-th/9804168

\title{
Comments on the Background Field Method in Harmonic Superspace: Non-holomorphic Corrections in $\mathrm{N}=4 \mathrm{SYM}$
}

\author{
Ioseph L. Buchbinder \\ Department of Theoretical Physics, Tomsk State Pedagogical University \\ Tomsk 634041, Russia \\ josephb@tspi.tomsk.su \\ Sergei M. Kuzenko \\ Department of Physics, Tomsk State University \\ Lenin Ave. 36, Tomsk 634050, Russia \\ kuzenko@phys.tsu.ru
}

\begin{abstract}
We analyse the one-loop effective action of $N=4$ SYM theory in the framework of the background field formalism in $N=2$ harmonic superspace. For the case of on-shell background $N=2$ vector multiplet we prove that the effective action is free of harmonic singularities. When the lowest $N=1$ superspace component of the $N=2$ vector multiplet is switched off, the effective action of $N=4$ SYM theory is shown to coincide with that obtained by Grisaru et al on the base of the $N=1$ background field method. We compute the leading non-holomorphic corrections to the $N=4 S U(2)$ SYM effective action.
\end{abstract}




\section{Introduction}

Harmonic superspace approach [1] is the only manifestly $N=2$ supersymmetric formalism developed which makes it possible to describe general $N=2$ super Yang-Mills theories in terms of unconstrained superfields and with explicitly realized authomorphism $S U(2)_{R}$ symmetry of the $N=2$ Poincaré superalgebra. The Feynman rules in harmonic superspace [2] have been successfully applied to compute the holomorphic corrections to the effective action of $N=2$ Maxwell multiplet coupled to the charged matter $q-$ hypermultiplet [3, 4] as well as the induced hypermultiplet self-coupling [5]. Recently we have developed the background field method in harmonic superspace [6] and applied it to rigorously prove the $N=2$ non-renormalization theorem [7]. The background field formalism along with the results of Ref. [3] allowed us to derive Seiberg's holomorphic action $\mathcal{F}(\mathcal{W})$ for $N=2 S U(2)$ SYM theory [8] for the first time directly in $N=2$ superspace (see Refs. [9, 10] for $N=1$ calculations). However, there appeared some puzzle with computing the leading non-holomorphic correction $\mathcal{H}(\mathcal{W}, \overline{\mathcal{W}})$, which we are going to describe a bit later.

In the Coulomb branch of $N=2 S U(2)$ SYM theory [11], the effective action $\Gamma[\mathcal{W}, \overline{\mathcal{W}}]$ is a functional of the Abelian $N=2$ strength $\mathcal{W}$ and its conjugate $\overline{\mathcal{W}}$. Assuming the validity of momentum expansion, one can present $\Gamma[\mathcal{W}, \overline{\mathcal{W}}]$ in the form

$$
\Gamma[\mathcal{W}, \overline{\mathcal{W}}]=\left(\int \mathrm{d}^{4} \theta \mathrm{d}^{4} \theta \mathcal{L}_{\text {eff }}^{(\mathrm{c})}+\text { c.c. }\right)+\int \mathrm{d}^{4} x \mathrm{~d}^{8} \theta \mathcal{L}_{\text {eff }}
$$

Here the chiral effective Lagrangian $\mathcal{L}_{\text {eff }}^{(\mathrm{c})}$ is a local function of $\mathcal{W}$ and its space-time derivatives, $\mathcal{L}_{\text {eff }}^{(\mathrm{c})}=\mathcal{F}(\mathcal{W})+\ldots$, and the higher-derivative effective Lagrangian $\mathcal{L}_{\text {eff }}$ is a real function of $\mathcal{W}, \overline{\mathcal{W}}$ and their covariant derivatives, $\mathcal{L}_{\text {eff }}=\mathcal{H}(\mathcal{W}, \overline{\mathcal{W}})+\ldots$ The possibility of non-nolomorphic quantum corrections $\mathcal{H}(\mathcal{W}, \overline{\mathcal{W}})$ was first pointed out in 12 . In the case of $N=4 \mathrm{SYM}$ theory realized in $N=2$ superspace, $\mathcal{H}(\mathcal{W}, \overline{\mathcal{W}})$ constitutes the leading quantum correction and should have the following structure [13] (see also [9])

$$
\mathcal{H}(\mathcal{W}, \overline{\mathcal{W}})=c \ln \frac{\mathcal{W}^{2}}{\Lambda^{2}} \ln \frac{\overline{\mathcal{W}}^{2}}{\Lambda^{2}}
$$

with some constant $c$. There are strong indications that in $N=4 \mathrm{SYM}$ theory $\mathcal{H}(\mathcal{W}, \overline{\mathcal{W}})$ perturbatively is a pure one-loop effect [13] (see also [7]), and nonperturbative corrections vanish [14]. The explicit value of $c$ was given in [15] to be the result of supergraph calculations with use of the $N=1$ background field formalism

$$
c=\frac{1}{4(4 \pi)^{2}} \text {. }
$$


Indirect calculations in projective superspace [16] led to the same value for $c$ (the authors of Ref. [16] computed in fact the low-energy hupermultiplet action, and then identified it with that corresponding to the vector multiplet) $t$. What happens in harmonic superspace?

It turns out that the one-loop supergraphs contributing to $\mathcal{H}(\mathcal{W}, \overline{\mathcal{W}})$ in the harmonic superspace approach contain coinciding harmonic singularities, that is harmonic distributions at coinciding points. The problem of coinciding harmonic singularities in the framework of harmonic supergraph Feynman rules was first discussed in [17]. Such singularities have no physical origin, in contrast to ultraviolet divergences. They can appear only at intermediate stages of calculation and should cancel each other in the final expressions for physical quantities. But it is clear that one should very carefully handle the relevant supergraphs in order to result in correct amplitudes. We should stress that the problem in field seems to be equally well characteristic of the Feynman rules in $N=2$ projective superspace [18], since the structure of projective propagators is very similar to that of harmonic ones. The origin of this problem is an infinite number of internal degrees of freedom associated with the bosonic internal coordinates.

To get rid of the one-loop coinciding harmonic singularities, in [6] we introduced, as is generally accepted in quantum field theory, some regularization of harmonic distributions. Unfortunately, this regularization proves to be unsuccessful; its use led us to the wrong conclusion $\mathcal{H}(\mathcal{W}, \overline{\mathcal{W}})=0$. In a sense, the situation in hand is similar to that with the wellknown supersymmetric regularization via dimensional reduction which leads to obstacles at higher loops. The harmonic regularization we used turned out to be improper already at the one-loop level 2 .

In the present paper we demonstrate that the one-loop coinciding harmonic singularities are easily factorized in the case with the background $N=2$ vector multiplet on-shell. Such a choice of background superfields is by no means critical, but it leads to a number of technical simplifications. When the $N=1$ chiral scalar part of the $N=2$ vector multiplet is switched off, we show that the effective action of $N=4$ SYM, which is derived in the framework of the background field formalism in $N=2$ harmonic superspace, coincides with that obtained on the base of $N=1$ background field formalism [19]. We also compute the leading non-holomorphic corrections (2) to the effective action of $N=4$

\footnotetext{
${ }^{1}$ The value for $c$ given in the first version of [16] had opposite sign as compared with (3).

${ }^{2}$ We would like to emphasize that the problem of coinciding harmonic singularities is associated only with perturbative calculations of the effective action and has no direct relation to the $N=2$ background field method itself.
} 
$S U(2)$ SYM theory and obtain the correct value of $c$ (3).

The paper is organized as follows. In section 2 we describe the factorization of harmonic singularities and obtain a useful representation for the effective action of $N=4$ SYM in terms of constrained $N=2$ superfields. In section 3 we fulfil the reduction to $N=1$ superfields and establish the fact that the two background field formalisms, in $N=2$ harmonic superspace and in $N=1$ superspace, lead to the same one-loop effective action for $N=4 \mathrm{SYM}$. In section 4 we compute $\mathcal{H}(\mathcal{W}, \overline{\mathcal{W}})$. This paper is as a direct continuation of our work [6]. The conventions we follow are those of Refs. [6, 7] with the only exclusion: here we denote the analytic subspace measure by $\mathrm{d} \zeta^{(-4)}$, instead of notation $\mathrm{d} \zeta^{(-4)} \mathrm{d} u$ used in [6, 7].

\section{Elimination of harmonic singularities}

According to the results of Ref. [6], the purely Yang-Mills part $\Gamma^{(1)}\left[V^{++}\right]$of the one-loop effective action $\Gamma^{(1)}$ in a general $N=2$ SYM theory is given by

$$
\begin{aligned}
\Gamma^{(1)}\left[V^{++}\right]= & \frac{\mathrm{i}}{2} \operatorname{Tr}_{(2,2)} \ln \widehat{\square}_{\lambda}-\frac{\mathrm{i}}{2} \operatorname{Tr}_{(4,0)} \ln \widehat{\triangle}_{\lambda} \\
& +\mathrm{i} \operatorname{Tr}_{R_{q}} \ln \left(\nabla^{++}\right)+\frac{\mathrm{i}}{2} \operatorname{Tr}_{R_{\omega}} \ln \left(\nabla^{++}\right)^{2}-\frac{\mathrm{i}}{2} \operatorname{Tr}_{a d} \ln \left(\nabla^{++}\right)^{2} .
\end{aligned}
$$

Here $\widehat{\square}$ is the analytic d'Alembertian which reads in the $\tau$-frame as follows

$$
\begin{aligned}
\widehat{\square}= & \mathcal{D}^{m} \mathcal{D}_{m}+\frac{\mathrm{i}}{2}\left(\mathcal{D}^{+\alpha} W\right) \mathcal{D}_{\alpha}^{-}+\frac{\mathrm{i}}{2}\left(\overline{\mathcal{D}}_{\dot{\alpha}}^{+} \bar{W}\right) \overline{\mathcal{D}}^{-\dot{\alpha}}-\frac{\mathrm{i}}{4}\left(\mathcal{D}^{+\alpha} \mathcal{D}_{\alpha}^{+} W\right) \mathcal{D}^{--} \\
& +\frac{\mathrm{i}}{8}\left[\mathcal{D}^{+\alpha}, \mathcal{D}_{\alpha}^{-}\right] W+\frac{1}{2}\{\bar{W}, W\}
\end{aligned}
$$

The contributions in the first line of eq. (4) come from the following path integrals

$$
\begin{aligned}
\left(\operatorname{Det}_{(2,2)} \widehat{\square}_{\lambda}\right)^{-1} & =\int \mathcal{D} v^{++} \mathcal{D} u^{++} \exp \left\{-\mathrm{i} \operatorname{tr} \int \mathrm{d} \zeta^{(-4)} v^{++} \widehat{\square}_{\lambda} u^{++}\right\} \\
\left(\operatorname{Det}_{(4,0)} \widehat{\square}_{\lambda}\right)^{-1} & =\int \mathcal{D} \rho^{(+4)} \mathcal{D} \sigma \exp \left\{-\mathrm{i} \operatorname{tr} \int \mathrm{d} \zeta^{(-4)} \rho^{(+4)} \widehat{\square}_{\lambda} \sigma\right\}
\end{aligned}
$$

over unconstrained bosonic analytic real superfields $v^{++}, u^{++}$and $\rho^{(+4)}, \sigma$. The contributions in the second line of eq. (4) correspond to the matter $q$-hypermultiplets (in a complex representation $R_{q}$ of the gauge group) and $\omega$-hupermultiplets (in a real representation $R_{\omega}$ ) as well as to the ghost superfields transforming in the adjoint representation. 
In the case of $N=4 \mathrm{SYM}$ theory realized in $N=2$ harmonic superspace [2] the matter sector is formed by a single $\omega$-hypermultiplet in the adjoint representation, hence the one-loop effective action (甘) turns into

$$
\Gamma_{N=4}^{(1)}=\frac{\mathrm{i}}{2} \operatorname{Tr}_{(2,2)} \ln \widehat{\square}_{\lambda}-\frac{\mathrm{i}}{2} \operatorname{Tr}(4,0) \ln \widehat{\square}_{\lambda} .
$$

A simple inspection of harmonic supergraphs shows that both contributions to $\Gamma_{N=4}^{(1)}$ contain harmonic singularities. On general grounds, the singular parts of $\operatorname{Tr}_{(2,2)} \ln \widehat{\square}_{\lambda}$ and $\operatorname{Tr}(4,0) \ln \widehat{\square}_{\lambda}$ should cancel each other. Complete factorization of harmonic singularities can be most simply established in the case when the background $N=2$ gauge superfield is chosen on-shell

$$
\mathcal{D}^{\alpha(i} \mathcal{D}_{\alpha}^{j)} W=0
$$

what we assume below. A more complete analysis will be given somewhere else. Under requirement (9) the operators $\mathcal{D}^{++}$and $\widehat{\square}$, which move every analytic superfield into analytic ones, commute as a consequence of the identity (given in the $\tau$-frame)

$$
\left[\mathcal{D}^{++}, \widehat{\square}\right] \Phi^{(q)}=\frac{\mathrm{i}}{4}(1-q)\left(\mathcal{D}^{+\alpha} \mathcal{D}_{\alpha}^{+} W\right) \Phi^{(q)}
$$

for an arbitrary analytic superfield $\Phi^{(q)}$ with $U(1)$ charge $q$.

Let us consider the following non-degenerate replacement of variables

$$
\begin{aligned}
v^{++} & =\mathcal{F}^{++}+\nabla^{++} \sigma \\
u^{++} & =\mathcal{G}^{++}+\nabla^{++} \int \mathrm{d} \tilde{\zeta}^{(-4)} \mathbf{G}^{(0,0)}(\zeta, \tilde{\zeta}) \rho^{(+4)}(\tilde{\zeta})
\end{aligned}
$$

where $v^{++}, u^{++}$and $\sigma, \rho^{(+4)}$ are unconstrained analytic real superfields, while the analytic real superfields $\mathcal{F}^{++}$and $\mathcal{G}^{++}$are constrained to be covariantly linear

$$
\nabla^{++} \mathcal{F}^{++}=0 \quad \nabla^{++} \mathcal{G}^{++}=0
$$

The Green's function $\mathbf{G}^{(0,0)}\left(\zeta_{1}, \zeta_{2}\right)$ is the Feynman propagator of $\omega$-hypermultiplet coupled to the $N=2$ gauge superfield. It satisfies the equation

$$
\left(\nabla_{1}^{++}\right)^{2} \mathbf{G}^{(0,0)}(1,2)=-\delta_{A}^{(4,0)}(1,2)
$$

and reads most simply in the $\tau$-frame [7]

$$
\mathbf{G}_{\tau}^{(0,0)}(1,2)=\frac{1}{\widehat{\square}_{1}}\left(\overrightarrow{\mathcal{D}_{1}^{+}}\right)^{4}\left\{\delta^{12}\left(z_{1}-z_{2}\right) \frac{\left(u_{1}^{-} u_{2}^{-}\right)}{\left(u_{1}^{+} u_{2}^{+}\right)^{3}}\right\}\left(\overleftarrow{\mathcal{D}_{2}^{+}}\right)^{4}
$$


We fulfil the replacement of variables (11) in the path integral

$$
1=\int \mathcal{D} v^{++} \mathcal{D} u^{++} \exp \left\{\mathrm{i} \operatorname{tr} \int \mathrm{d} \zeta^{(-4)} v^{++} u^{++}\right\}
$$

Denoting by $J$ the corresponding Jacobian, we get

$$
\begin{aligned}
1= & J \int \mathcal{D} \rho^{(+4)} \mathcal{D} \sigma \exp \left\{\mathrm{i} \operatorname{tr} \int \mathrm{d} \zeta^{(-4)} \rho^{(+4)} \sigma\right\} \\
& \times \int \mathcal{D} \mathcal{F}^{++} \mathcal{D} \mathcal{G}^{++} \exp \left\{\mathrm{i} \operatorname{tr} \int \mathrm{d} \zeta^{(-4)} \mathcal{F}^{++} \mathcal{G}^{++}\right\} \\
= & J \int \mathcal{D} \mathcal{F}^{++} \mathcal{D} \mathcal{G}^{++} \exp \left\{\mathrm{i} \operatorname{tr} \int \mathrm{d} \zeta^{(-4)} \mathcal{F}^{++} \mathcal{G}^{++}\right\} .
\end{aligned}
$$

Now, we fulfil the same replacement of variables in (6)

$$
\begin{aligned}
\left(\operatorname{Det}_{(2,2)} \widehat{\square}_{\lambda}\right)^{-1}= & J \int \mathcal{D} \rho^{(+4)} \mathcal{D} \sigma \exp \left\{-\mathrm{i} \operatorname{tr} \int \mathrm{d} \zeta^{(-4)} \rho^{(+4)} \widehat{\square}_{\lambda} \sigma\right\} \\
& \times \int \mathcal{D} \mathcal{F}^{++} \mathcal{D} \mathcal{G}^{++} \exp \left\{-\mathrm{i} \operatorname{tr} \int \mathrm{d} \zeta^{(-4)} \mathcal{F}^{++} \widehat{\square}_{\lambda} \mathcal{G}^{++}\right\} .
\end{aligned}
$$

Expressing here $J$ as in eq. (16) and making use of (7), we then result in

$$
\exp \left\{2 \mathrm{i} \Gamma_{N=4}^{(1)}\right\}=\frac{\int \mathcal{D} \mathcal{F}^{++} \mathcal{D} \mathcal{G}^{++} \exp \left\{-\mathrm{i} \operatorname{tr} \int \mathrm{d} \zeta^{(-4)} \mathcal{F}^{++} \widehat{\square}_{\lambda} \mathcal{G}^{++}\right\}}{\int \mathcal{D} \mathcal{F}^{++} \mathcal{D} \mathcal{G}^{++} \exp \left\{\mathrm{i} \operatorname{tr} \int \mathrm{d} \zeta^{(-4)} \mathcal{F}^{++} \mathcal{G}^{++}\right\}}
$$

It is not difficult to obtain another representation for the effective action

$$
\exp \left\{\mathrm{i} \Gamma_{N=4}^{(1)}\right\}=\frac{\int \mathcal{D} \mathcal{F}^{++} \exp \left\{-\frac{\mathrm{i}}{2} \operatorname{tr} \int \mathrm{d} \zeta^{(-4)} \mathcal{F}^{++} \widehat{\square}_{\lambda} \mathcal{F}^{++}\right\}}{\int \mathcal{D} \mathcal{F}^{++} \exp \left\{\frac{\mathrm{i}}{2} \operatorname{tr} \int \mathrm{d} \zeta^{(-4)} \mathcal{F}^{++} \mathcal{F}^{++}\right\}}
$$

which will be the starting point for our further analysis.

Both functional integrals in the r.h.s. of (19) are carried out over constrained analytic superfields depending on the background gauge superfield. In particular, the functional measure $\mathcal{D} \mathcal{F}^{++}$depends nontrivially on the gauge superfield. But the relevant constraint

$$
\nabla^{++} \mathcal{F}^{++}=D^{++} \mathcal{F}^{++}+\mathrm{i}\left[V^{++}, \mathcal{F}^{++}\right]=0
$$

can be solved in terms of a $V^{++}$independent linear analytic superfield $\mathrm{F}^{++}$

$$
D^{++} F^{++}=0
$$

as a power series in $V^{++}$

$$
\mathcal{F}^{++}=F^{++}+\sum_{n=1}^{\infty} A^{n} F^{++}
$$


where the operator $A$ transforms any analytic superfield $\phi^{++}(\zeta)$ into an analytic one and is defined by

$$
A \phi^{++}(\zeta)=\mathrm{i} D^{++} \int \mathrm{d} \tilde{\zeta}^{(-4)} \mathrm{G}^{(0,0)}(\zeta, \tilde{\zeta})\left[V^{++}(\tilde{\zeta}), \phi^{++}(\tilde{\zeta})\right]
$$

Here $\mathrm{G}^{(0,0)}(1,2)$ is the free $\omega$-hypermultiplet propagator [2] and it can be obtained from (14) by switching off the background gauge superfield. Of course, the functional integration measures $\mathcal{D} \mathcal{F}^{++}$and $\mathcal{D} F^{++}$are related by some $V^{++}$dependent Jacobian, but the latter drops out of the r.h.s. of (19). Therefore, we can simply replace $\mathcal{D F}^{++}$in the r.h.s. of (19) by $\mathcal{D} F^{++}$and then pertubatively compute both functional integrals by properly defining the relevant free propagators. This is a simple task after heroic efforts undertaken by the projective superspace group [18] on working out the propagators of constrained superfields. For example, the propagator corresponding to a free tensor multiplet with action 20

$$
S=\frac{1}{2} \int \mathrm{d} \zeta^{(-4)}\left(F^{++}\right)^{2}
$$

reads

$$
\begin{aligned}
& <F^{++}(1) F^{++}(2)>=\mathrm{i} \Pi^{(2,2)}(1,2) \\
& \Pi^{(2,2)}(1,2)=-\frac{\left(D_{1}^{+}\right)^{4}\left(D_{2}^{+}\right)^{4}}{\square_{1}} \frac{1}{\left(u_{1}^{+} u_{2}^{+}\right)^{2}} \delta^{12}\left(z_{1}-z_{2}\right)
\end{aligned}
$$

with $\Pi^{(2,2)}(1,2)$ the projector operator [2] for the analytic superfields constrained by (21).

The main advantage of representation $(19)$ is that it contains no harmonic singularities. The integration in (19) is in fact carried out over ordinary $N=2$ constrained superfields coupled to the super Yang-Mills multiplet. This becomes obvious in the $\tau$-frame in which $\mathcal{F}^{++}$reads

$$
\mathcal{F}_{\tau}^{++}=\mathcal{F}^{i j}(z) u_{i}^{+} u_{j}^{+}
$$

where the $u$-independent isovector $\mathcal{F}^{i j}$ is real

$$
\overline{\mathcal{F} i j}=\mathcal{F}_{i j}
$$

and satisfies the constraints

$$
\mathcal{D}_{\alpha}^{(i} \mathcal{F}^{j k)}=\overline{\mathcal{D}}_{\dot{\alpha}}^{(i} \mathcal{F}^{j k)}=0
$$

Because of (9), the operator $\square$ moves the space of such superfields into itself

$$
\begin{aligned}
\overparen{\square \mathcal{F}^{i j}=} & \left(\mathcal{D}^{a} \mathcal{D}_{a}+\frac{1}{2}\{W, \bar{W}\}\right) \mathcal{F}^{i j} \\
& +\frac{\mathrm{i}}{3}\left(\mathcal{D}^{\alpha(i} W\right) \cdot \mathcal{D}_{\alpha|k|} \mathcal{F}^{j) k}+\frac{\mathrm{i}}{3} \overline{\mathcal{D}}_{\dot{\alpha}}^{(i} \bar{W} \cdot \overline{\mathcal{D}}_{|k|}^{\dot{\alpha}} \mathcal{F}^{j) k}
\end{aligned}
$$

At the same time the representation (19) allows us to make a simple reduction to $N=1$ superspace. 


\section{Reduction to $\mathbf{N}=1$ superfields}

We introduce the Grassmann coordinates of $N=1$ superspace $\left(\theta^{\alpha}, \bar{\theta}_{\dot{\alpha}}\right)$ as part of those $\left(\theta_{i}^{\alpha}, \bar{\theta}_{\dot{\alpha}}^{j}\right)$ parametrizing its $N=2$ extension

$$
\theta^{\alpha}=\theta_{1}^{\alpha} \quad \bar{\theta}_{\dot{\alpha}}=\bar{\theta}_{\dot{\alpha}}^{1}
$$

and define $N=1$ projections of $N=2$ superfields by the standard rule

$$
U\left|=U\left(x^{m}, \theta_{i}^{\alpha}, \bar{\theta}_{\dot{\alpha}}^{j}\right)\right|_{\theta_{2}=\bar{\theta}^{2}=0}
$$

The $N=1$ gauge covariant derivatives are

$$
\mathcal{D}_{\alpha}=\mathcal{D}_{\alpha}^{1}\left|=D_{\alpha}^{1}+\mathrm{i} \mathcal{A}_{\alpha}^{1}\right| \quad \overline{\mathcal{D}}^{\dot{\alpha}}=\overline{\mathcal{D}}_{1}^{\dot{\alpha}}\left|=\bar{D}_{1}^{\dot{\alpha}}+\mathrm{i} \overline{\mathcal{A}}_{1}^{\dot{\alpha}}\right|
$$

The covariantly chiral $N=2$ strength $W$ leads to the two $N=1$ superfields

$$
\begin{aligned}
\Phi=W \mid & \overline{\mathcal{D}}_{\dot{\alpha}} \Phi=0 \\
2 \mathrm{i} W_{\alpha}=\mathcal{D}_{\alpha}^{2} W \mid & \overline{\mathcal{D}}_{\dot{\alpha}} W_{\alpha}=0
\end{aligned}
$$

which are covariantly chiral, and $W_{\alpha}$ satisfies the Bianchi identity

$$
\mathcal{D}^{\alpha} W_{\alpha}=\overline{\mathcal{D}}_{\dot{\alpha}} \bar{W}^{\dot{\alpha}}
$$

The algebra of $N=1$ derivatives reads

$$
\begin{array}{ll}
\left\{\mathcal{D}_{\alpha}, \overline{\mathcal{D}}_{\dot{\alpha}}\right\}=-2 \mathrm{i} \mathcal{D}_{\alpha \dot{\alpha}} & \left\{\mathcal{D}_{\alpha}, \mathcal{D}_{\beta}\right\}=\left\{\overline{\mathcal{D}}_{\dot{\alpha}}, \overline{\mathcal{D}}_{\dot{\beta}}\right\}=0 \\
{\left[\mathcal{D}_{\alpha \dot{\alpha}}, \mathcal{D}_{\beta}\right]=-2 \mathrm{i} \varepsilon_{\alpha \beta} \bar{W}_{\dot{\alpha}}} & {\left[\mathcal{D}_{\alpha \dot{\alpha}}, \overline{\mathcal{D}}_{\dot{\beta}}\right]=-2 \mathrm{i} \varepsilon_{\dot{\alpha} \dot{\beta}} W_{\alpha}}
\end{array}
$$

One of the $N=1$ counterparts of requirement (9) is

$$
\mathcal{D}^{\alpha} W_{\alpha}=[\bar{\Phi}, \Phi]
$$

The $N=1$ projections of the superfield $\mathcal{F}^{i j}$ constrained by eqs. (27) and (28) are

$$
\Psi=\mathcal{F}^{22}\left|\quad \bar{\Psi}=\mathcal{F}^{11}\right| \quad F=\bar{F}=-2 \mathrm{i} \mathcal{F}^{12} \mid
$$

and satisfy the constraints

$$
\overline{\mathcal{D}}_{\dot{\alpha}} \Psi=0 \quad-\frac{1}{4} \overline{\mathcal{D}}^{2} F+[\Phi, \Psi]=0
$$


Therefore, $\Psi$ is a covariantly chiral superfield, while the real superfield $F$ is subject to a modified linear constraint.

To reduce the actions arising in the r.h.s. of (19) to $N=1$ superfields, we can go on as follows. Let $L^{(+4)}(\zeta)$ be a gauge invariant real analytic superfield constrained by

$$
D^{++} L^{(+4)}=0
$$

Such a superfield can be represented in the form

$$
L^{(+4)}(\zeta)=L^{i j k l}(z) u_{i}^{+} u_{j}^{+} u_{k}^{+} u_{l}^{+}
$$

where the $u$-independent superfield $L^{i j k l}$ satisfies the constraints

$$
D_{\alpha}^{\left(i_{1}\right.} L^{\left.i_{2} \cdots i_{5}\right)}=\bar{D}_{\dot{\alpha}}^{\left(i_{1}\right.} L^{\left.i_{2} \cdots i_{5}\right)}=0
$$

These constraints imply

$$
\int \mathrm{d} \zeta^{(-4)} L^{(+4)}=6 \int \mathrm{d}^{8} z L^{1122} \mid \quad \mathrm{d}^{8} z=\mathrm{d}^{4} x \mathrm{~d}^{2} \theta \mathrm{d}^{2} \bar{\theta}
$$

with $\mathrm{d}^{8} z$ being the full $N=1$ superspace measure. In the role of $L^{(+4)}$ we choose

$$
L^{(+4)}=\operatorname{tr}\left(\mathcal{F}^{++} \mathcal{G}^{++}\right)=\operatorname{tr}\left(\mathcal{F}_{\tau}^{++} \mathcal{G}_{\tau}^{++}\right)
$$

with analytic superfields $\mathcal{F}^{++}$and $\mathcal{G}^{++}$constrained as in (12). Then we get

$$
\operatorname{tr} \int \mathrm{d} \zeta^{(-4)} \mathcal{F}^{++} \mathcal{G}^{++}=\operatorname{tr} \int \mathrm{d}^{8} z\left(\mathcal{F}^{11}\left|\mathcal{G}^{22}\right|+\mathcal{G}^{11}\left|\mathcal{F}^{22}\right|+4 \mathcal{F}^{12}\left|\mathcal{G}^{12}\right|\right)
$$

To compare our $N=2$ superspace results for the one-loop effective action of $N=4$ super Yang-Mills theory with those derived in $N=1$ superspace [19], we switch off the lowest $N=1$ superspace component of the $N=2$ vector multiplet by setting

$$
\Phi=0
$$

Then eq. (29) takes the form

$$
\left(\widehat{\square \mathcal{F}^{i j}}\right)\left|=\widetilde{\square} \mathcal{F}^{i j}\right| \quad \tilde{\square}=\mathcal{D}^{a} \mathcal{D}_{a}-W^{\alpha} \mathcal{D}_{\alpha}+\bar{W}_{\dot{\alpha}} \overline{\mathcal{D}}^{\dot{\alpha}}
$$

As is seen, the operator $\tilde{\square}$ does not mix the components of $\mathcal{F}^{i j} \mid$. It is not surprising, but nevertheless very important that $\tilde{\square}$ is exactly the operator which enters the quadratic 
part of quantum gauge superfield action in the $N=1$ background field approach 19. Now, eq. (19) turns into

$$
\exp \left\{\mathrm{i} \Gamma_{N=4}^{(1)}\right\}=\frac{\int \mathcal{D} \bar{\Psi} \mathcal{D} \Psi \mathcal{D} F \exp \left\{\mathrm{i} \operatorname{tr} \int \mathrm{d}^{8} z\left(-\bar{\Psi} \tilde{\square} \Psi+\frac{1}{2} F \widetilde{\square} F\right)\right\}}{\int \mathcal{D} \bar{\Psi} \mathcal{D} \Psi \mathcal{D} F \exp \left\{\mathrm{i} \operatorname{tr} \int \mathrm{d}^{8} z\left(\bar{\Psi} \Psi-\frac{1}{2} F^{2}\right)\right\}} .
$$

It should be remarked that here $F$ is a covariantly linear superfield, as a consequence of eqs. (38) and (45).

Let us recall the path-integral representation for $\Gamma_{N=4}^{(1)}$ derived in the framework of $N=1$ background field method 19

$$
\exp \left\{\mathrm{i} \Gamma_{N=4}^{(1)}\right\}=\int \mathcal{D} U \exp \left\{\frac{\mathrm{i}}{2} \operatorname{tr} \int \mathrm{d}^{8} z U \widetilde{\square} U\right\}
$$

where the integration variable $U$ is an unconstrained real superfield. It is not difficult to see that the representations (47) and (48) are equivalent. Really, because of eqs. (36) and (45) we have a well defined and gauge covariant decomposition of $U$ into a sum of chiral-antichiral and linear parts

$$
U=\Psi+\bar{\Psi}+F \quad \overline{\mathcal{D}}_{\dot{\alpha}} \Psi=0 \quad \overline{\mathcal{D}}^{2} F=0 .
$$

Jacobian $J$ of such a replacement of variables can be read off from

$$
\begin{aligned}
1 & =\int \mathcal{D} U \exp \left\{\frac{\mathrm{i}}{2} \operatorname{tr} \int \mathrm{d}^{8} z U^{2}\right\} \\
& =J \int \mathcal{D} \bar{\Psi} \mathcal{D} \Psi \mathcal{D} F \exp \left\{\mathrm{i} \operatorname{tr} \int \mathrm{d}^{8} z\left(\bar{\Psi} \Psi+\frac{1}{2} F^{2}\right)\right\} .
\end{aligned}
$$

If we fulfil the replacement of variables (49) in (48) and represent the corresponding Jacobian as in the relation just obtained, we will end up with (47).

Another interesting representation for $\Gamma_{N=4}^{(1)}$ can be obtained when $\Phi$ is covariantly constant and lies along a flat direction of the $N=2 \mathrm{SYM}$ potential

$$
\mathcal{D}_{\alpha} \Phi=0 \quad[\bar{\Phi}, \Phi]=0
$$

what is consistent only if

$$
\left[W_{\alpha}, \Phi\right]=\left[\bar{W}_{\dot{\alpha}}, \Phi\right]=0
$$

In this case we have

$$
\left(\widehat{\square \mathcal{F}^{i j}}\right)\left|=\Delta \mathcal{F}^{i j}\right| \quad \Delta=\mathcal{D}^{a} \mathcal{D}_{a}-W^{\alpha} \mathcal{D}_{\alpha}+\bar{W}_{\dot{\alpha}} \overline{\mathcal{D}}^{\dot{\alpha}}+\frac{1}{2}\{\bar{\Phi}, \Phi\} .
$$


The background gauge freedom can be used to make $\Phi$ constant. Then we stay with unbroken gauge transformations leaving $\Phi$ invariant. Let us for simplicity consider $S U(2)$ in the role of gauge group (with the generators $\tau^{\underline{a}}=\frac{1}{\sqrt{2}} \sigma^{\underline{a}}$ ) and choose the $N=2$ strength $W$ in the $z$-direction

$$
W=W^{\underline{3}} \tau^{\underline{3}} \equiv \mathcal{W} \tau^{\underline{3}} \quad \Phi \equiv \phi \tau^{\underline{3}} \quad W_{\alpha} \equiv \mathcal{W}_{\alpha} \tau^{\underline{3}}
$$

Since only the $U(1)$ subgroup generated by $\tau^{\underline{3}}$ is gauged, the $z$-components of all quantum superfields $F^{\underline{a}}, \Psi^{\underline{a}}$ and $\bar{\Psi}^{\underline{a}}$ do not interact with the background superfields and, hence, completely decouple. It is useful to combine the rest components into ones having definite charge with respect to $\tau^{\underline{3}}$

$$
\begin{aligned}
V & =F^{\underline{1}}-\mathrm{i} F^{\underline{2}} & & \tau^{\underline{3}} V=e V \\
\Psi_{ \pm} & =\Psi^{\underline{1}} \mp \mathrm{i} \Psi^{2} & & \tau^{\underline{3}} \Psi_{ \pm}= \pm e \Psi_{ \pm}
\end{aligned}
$$

with $e=\sqrt{2}$. Now, eq. (38) tells us

$$
\frac{1}{4} \overline{\mathcal{D}}^{2} V=\phi e \Psi_{+} \quad \frac{1}{4} \overline{\mathcal{D}}^{2} \bar{V}=-\phi e \Psi_{-} .
$$

These relations can be treated as the definition of chiral superfields $\Psi_{+}$and $\Psi_{-}$in terms of an unconstrained complex scalar superfield $V$ and its conjugate. As a result, eq. (19) becomes

$$
\exp \left\{\mathrm{i} \Gamma_{N=4}^{(1)}\right\}=\frac{\int \mathcal{D} \bar{V} \mathcal{D} V \exp \left\{-\frac{\mathrm{i}}{2} \operatorname{tr} \int \mathrm{d}^{8} z \bar{V} \mathbf{B} \Delta V\right\}}{\int \mathcal{D} \bar{V} \mathcal{D} V \exp \left\{\frac{\mathrm{i}}{2} \operatorname{tr} \int \mathrm{d}^{8} z \bar{V} \mathbf{B} V\right\}}
$$

where $\mathbf{B}$ denotes the following non-singular operator

$$
\mathbf{B}=\frac{1}{16}\left\{\overline{\mathcal{D}}^{2}, \mathcal{D}^{2}\right\}-e^{2}|\phi|^{2}
$$

Since $V$ is unconstrained, from here we immediately get

$$
\exp \left\{\mathrm{i} \Gamma_{N=4}^{(1)}\right\}=\int \mathcal{D} \bar{V} \mathcal{D} V \exp \left\{\frac{\mathrm{i}}{2} \operatorname{tr} \int \mathrm{d}^{8} z \bar{V} \Delta V\right\}
$$

where $\Delta$ now reads

$$
\Delta=\mathcal{D}^{a} \mathcal{D}_{a}-e \mathcal{W}^{\alpha} \mathcal{D}_{\alpha}+e \overline{\mathcal{W}}_{\dot{\alpha}} \overline{\mathcal{D}}^{\dot{\alpha}}+e^{2}|\phi|^{2}
$$

The structure of operator $\Delta$ is such that $\Gamma_{N=4}^{(1)}$ can be computed in the framework of the $N=1$ superfield Schwinger-DeWitt technique [21]. 


\section{Non-holomorphic corrections in $\mathrm{N}=4 \mathrm{SYM}$}

In the present we compute the leading non-holomorphic contribution to the one-loop effective action of $N=4 S U(2)$ super Yang-Mills theory

$$
\Gamma=\int \mathrm{d}^{12} z \mathcal{H}(\mathcal{W}, \overline{\mathcal{W}})
$$

where the Abelian $N=2$ strength $\mathcal{W}$ corresponds to the unbroken $U(1)$ subgroup of $S U(2)$ in the Coulomb branch. $\Gamma$ can be readily reduced to $N=1$ superfields. Introducing the $N=1$ projections of $\mathcal{W}$

$$
\phi=\mathcal{W} \mid \quad 2 \mathrm{i} \mathcal{W}_{\alpha}=\mathcal{D}_{\alpha}^{2} \mathcal{W}
$$

one gets (see Refs. [9, 12] for more detail)

$$
\Gamma=\int \mathrm{d}^{8} z \mathcal{W}^{\alpha} \mathcal{W}_{\alpha} \overline{\mathcal{W}}_{\dot{\alpha}} \overline{\mathcal{W}}^{\dot{\alpha}} \frac{\partial^{4} \mathcal{H}(\phi, \bar{\phi})}{\partial \phi^{2} \partial \bar{\phi}^{2}}+\cdots
$$

where the dots mean the terms of third and lower orders in $\mathcal{W}_{\alpha}$ and $\overline{\mathcal{W}}_{\dot{\alpha}}$. To compute the structure present here, it is sufficient to evaluate $\Gamma_{N=4}^{(1)}$ for constant $\phi$ and $\mathcal{W}_{\alpha}$, and such a background is consistent with our restriction (9) used throughout the present paper.

In accordance with eqs. (59) and (60), we have

$$
\Gamma_{N=4}^{(1)}=-\mathrm{i} \int_{0}^{\infty} \frac{\mathrm{d} s}{s} \mathrm{e}^{-\mathrm{i}\left(e^{2}|\phi|^{2}-\mathrm{i} \varepsilon\right) s} \int \mathrm{d}^{8} z \mathcal{U}(z, z \mid s)
$$

where $\mathcal{U}\left(z, z^{\prime} \mid s\right)$ denotes the Schwinger's kernel [21]

$$
\mathcal{U}\left(z, z^{\prime} \mid s\right)=\exp \left\{-\mathrm{i} s\left(\mathcal{D}^{a} \mathcal{D}_{a}-e \mathcal{W}^{\alpha} \mathcal{D}_{\alpha}+e \overline{\mathcal{W}}_{\dot{\alpha}} \overline{\mathcal{D}}^{\dot{\alpha}}\right)\right\} \delta^{8}\left(z-z^{\prime}\right)
$$

For computing $\Gamma$ given by eq. (63) it is sufficient to use the approximation

$$
\mathcal{U}\left(z, z^{\prime} \mid s\right) \approx \exp \left\{\operatorname{is}\left(e \mathcal{W}^{\alpha} D_{\alpha}-e \overline{\mathcal{W}}_{\dot{\alpha}} \bar{D}^{\dot{\alpha}}\right)\right\} \mathcal{U}_{0}\left(z, z^{\prime} \mid s\right)
$$

with $\mathcal{U}_{0}\left(z, z^{\prime} \mid s\right)$ the free Schwinger's kernel 21]

$$
\mathcal{U}_{0}\left(z, z^{\prime} \mid s\right)=\mathrm{e}^{-\mathrm{i} s \partial^{a} \partial_{a}} \delta^{8}\left(z-z^{\prime}\right)=\frac{\mathrm{i}}{(4 \pi \mathrm{i} s)^{2}} \delta^{4}\left(\theta-\theta^{\prime}\right) \mathrm{e}^{-\mathrm{i}\left(x-x^{\prime}\right)^{2} / 4 s} .
$$

Using the identity

$$
\frac{1}{16} D^{2} \bar{D}^{2} \delta^{4}\left(\theta-\theta^{\prime}\right)=1
$$


we obtain

$$
\begin{aligned}
\Gamma_{N=4}^{(1)} & \approx \frac{e^{4}}{(4 \pi)^{2}} \int \mathrm{d}^{8} z \mathcal{W}^{\alpha} \mathcal{W}_{\alpha} \overline{\mathcal{W}}_{\dot{\alpha}} \overline{\mathcal{W}}^{\dot{\alpha}} \int_{0}^{\infty} \mathrm{d} s s \mathrm{e}^{-s e^{2}|\phi|^{2}} \\
& =\frac{1}{(4 \pi)^{2}} \int \mathrm{d}^{8} z \mathcal{W}^{\alpha} \mathcal{W}_{\alpha} \overline{\mathcal{W}}_{\dot{\alpha}} \overline{\mathcal{W}}^{\dot{\alpha}} \frac{1}{\phi^{2} \bar{\phi}^{2}}
\end{aligned}
$$

where all terms involving derivatives of $\mathcal{W}_{\alpha}$ and $\overline{\mathcal{W}}_{\dot{\alpha}}$ have been omitted. Comparing the relation obtained with (63), we get

$$
\frac{\partial^{4} \mathcal{H}(\mathcal{W}, \overline{\mathcal{W}})}{\partial \mathcal{W}^{2} \partial \overline{\mathcal{W}}^{2}}=\frac{1}{(4 \pi)^{2}} \frac{1}{\mathcal{W}^{2} \overline{\mathcal{W}}^{2}}
$$

A general solution of this equation (modulo purely holomorphic terms) reads

$$
\begin{aligned}
\mathcal{H}(\mathcal{W}, \overline{\mathcal{W}}) & =\frac{1}{(4 \pi)^{2}} \ln \frac{\mathcal{W}}{\Lambda} \ln \frac{\overline{\mathcal{W}}}{\Lambda} \\
& +\mathcal{W} \bar{f}(\overline{\mathcal{W}})+f(\mathcal{W}) \overline{\mathcal{W}}
\end{aligned}
$$

with some scale $\Lambda$ and some holomorphic function $f(\mathcal{W})$. The structures in the second line are in conflict with the scale and chiral invariances of $N=4 \mathrm{SYM}$ theory. Moreover, all contributions to $\mathcal{H}(\mathcal{W}, \overline{\mathcal{W}})$ coming from the $N=2$ supergraphs involve equal powers of $\mathcal{W}$ and $\overline{\mathcal{W}}$. Therefore, we conclude

$$
\mathcal{H}(\mathcal{W}, \overline{\mathcal{W}})=\frac{1}{(4 \pi)^{2}} \ln \frac{\mathcal{W}}{\Lambda} \ln \frac{\overline{\mathcal{W}}}{\Lambda}=\frac{1}{4(4 \pi)^{2}} \ln \frac{\mathcal{W}^{2}}{\Lambda^{2}} \ln \frac{\overline{\mathcal{W}}^{2}}{\Lambda^{2}} .
$$

Our final expression for $\Gamma$ coincides with the result of $N=1$ calculations announced in [15] as well as with that obtained by indirect methods in projective superspace [16]. The overall sign for $\mathcal{H}(\mathcal{W}, \overline{\mathcal{W}})$ can be readily determined, if we compare the coefficient for $(\mathcal{W} \overline{\mathcal{W}})^{2}$ in a power series expansion of $\mathcal{H}(\mathcal{W}, \overline{\mathcal{W}})$, with respect to some point $\mathcal{W}_{0}$, with similar $N=1$ quantum corrections quadric in $\mathcal{W}_{\alpha}$ and $\overline{\mathcal{W}}_{\dot{\alpha}}$ which were found in Superspace [22]. In a separate paper we are going to compute $\mathcal{H}(\mathcal{W}, \overline{\mathcal{W}})$ directly in $N=2$ harmonic superspace and without any restrictions on the background $N=2$ gauge superfield.

Acknowledgements. The authors are grateful to E.I. Buchbinder, M.T. Grisaru, E.A. Ivanov, B.A. Ovrut and E. Sokatchev for valuable discussions. We acknowledge a partial support from RFBR grant, project No 96-02-1607; RFBR-DFG grant, project No 96-0200180; INTAS grant, INTAS-96-0308; grant in the field of fundamental natural sciences from Ministry of General and Professional Education of Russian Federation. 


\section{References}

[1] A. Galperin, E. Ivanov, S. Kalitzin, V. Ogievetsky and E. Sokatchev, Class. Quant. Grav. 1 (1984) 469.

[2] A. Galperin, E. Ivanov, V. Ogievetsky and E. Sokatchev, Class. Quant. Grav. 2 (1985) 601; 617.

[3] I.L. Buchbinder, E.I. Buchbinder, E.A. Ivanov, S.M. Kuzenko and B.A. Ovrut, Phys. Lett. B 412 (1997) 309.

[4] E.I. Buchbinder, I.L. Buchbinder, E.A. Ivanov and S.M. Kuzenko, Mod. Phys. Lett. A 13 (1998) 1071.

[5] E.A. Ivanov, S.V. Ketov, B.M. Zupnik, Nucl. Phys. B 509 (1997) 53.

[6] I.L. Buchbinder, E.I. Buchbinder, S.M. Kuzenko and B.A. Ovrut, Phys. Lett. B 417 (1998) 61.

[7] I.L. Buchbinder, S.M. Kuzenko and B.A. Ovrut, hep-th 9710142.

[8] N. Seiberg, Phys. Lett. B 206 (1988) 75.

[9] B. de Wit, M.T. Grisaru and M. Roček, Phys. Lett. B 347 (1996) 297.

[10] A. Pickering and P. West, Phys. Lett. B 383 (1996) 54; M.T. Grisaru, M. Roček and U. von Unge, Phys. Lett. B 383 (1996) 415; U. Lindström, F. Gonzalez-Rey, M. Roček and R. von Unge, Phys. Lett. B 388 (1996) 581; A. De Giovanni, M.T. Grisaru, M. Roček, R. von Unge and D. Zanon, Phys. Lett. B 409 (1997) 251.

[11] N. Seiberg and E. Witten, Nucl. Phys. B 426 (1994) 19; B 430 (1994) 485.

[12] M. Henningson, Nucl. Phys. B 458 (1996) 445.

[13] M. Dine and N. Seiberg, Phys. Lett. B 409 (1997) 239.

[14] N. Dorey, V.V. Khoze, M.P. Mattis, J. Slater and W.A. Weir, Phys. Lett. B 408 (1997) 213; D. Bellisai, F. Fucito, M. Matone and G. Travaglini, Phys. Rev. D 56 (1997) 5218.

[15] V. Periwal and R. von Unge, hep-th/9801121.

[16] F. Gonzalez-Rey and M. Roček, hep-th/9804010. 
[17] A. Galperin, Nguyen Anh Ky and E. Sokatchev, Mod. Phys. Lett. A 2 (1987) 33.

[18] F. Gonzalez-Rey, M. Roček, S. Wiles, U. Lindström and R. von Unge, hepth/9710250; F. Gonzalez-Rey and R. von Unge, hep-th/9711135; F. Gonzalez-Rey, hep-th/9712128.

[19] M.T. Grisaru, W. Siegel and M. Roček, Nucl. Phys. B 159 (1979) 429.

[20] A. Galperin, E. Ivanov and V. Ogievetsky, Nucl. Phys. B 282 (1987) 74.

[21] I.L. Buchbinder and S.M. Kuzenko, Ideas and Methods of Supersymmetry and Supergravity, IOP Publ., Bristol and Philadelphia (1995).

[22] S.J. Gates, M.T. Grisaru, M. Roček and W. Siegel, Superspace, Benjamin-Cummings, Reading, MA (1983) pp. 390, 391. 